\title{
Note sur « mot à mot »
}

Jean-Yves Laurichesse

\section{OpenEdition}

Journals

Édition électronique

URL : http://journals.openedition.org/ccs/630

DOI : $10.4000 /$ ccs. 630

ISSN : 2558-782X

\section{Éditeur :}

Presses universitaires de Rennes, Association des lecteurs de Claude Simon

\section{Édition imprimée}

Date de publication : 31 décembre 2009

Pagination : 17-23

ISBN : 9782354120580

ISSN : $1774-9425$

\section{Référence électronique}

Jean-Yves Laurichesse, « Note sur « mot à mot » », Cahiers Claude Simon [En ligne], 5 | 2009, mis en ligne le 21 septembre 2017, consulté le 15 septembre 2020. URL : http://journals.openedition.org/ccs/ 630

Cahiers Claude Simon 


\section{Note sur « Mot à mot"}

" Mot à mot», publié dans les Lettres nouvelles le 8 avril 1959, est le troisième texte donné par Claude Simon à la revue dirigée par Maurice Nadeau ${ }^{1}$. Depuis le mois de mars, elle est devenue hebdomadaire, mais ne le restera que jusqu'en décembre, ce rythme s'avérant trop difficile à tenir ${ }^{2}$. Simon a publié le mois précédent "Cendre " dans la Revue de Paris'. Après cette revue à l'orientation plus " classique », il revient à la " modernité " avec les Lettres nouvelles. De toute évidence, il ne souhaite s'enfermer dans aucune chapelle politique ou littéraire, ce qui le conduira à publier aussi bien dans les Lettres françaises ou Tel quel que dans la Nouvelle Revue Française $^{4}$.

Sur la page de couverture du numéro sont mis en exergue, sous l'intitulé « cette semaine », deux noms, celui de Claude Simon et, en caractères plus gros, celui d'Alberto Giacometti (pour un entretien avec Georges Charbonnier). Le texte d'ouverture est dû à Bernard

${ }^{1}$ Cf. Jean-Yves Laurichesse, "Note sur "Matériaux de construction" ", Cahiers Claude Simon, $\mathrm{n}^{\circ}$ 4, 2008, p. 19.

2 Cf. Bernard Pingaud, Une tâche sans fin. Mémoires, Seuil, 2009, p. 106 et sq.

${ }^{3}$ Ce texte a été réédité dans le numéro 2 des Cahiers Claude Simon (2006).

${ }^{4} \mathrm{Cf}$. la bibliographie des «Textes et fragments " élaborée par Alastair Duncan et Jean Duffy (CE, 1554-1558). 
Pingaud, membre du comité de rédaction. Il a pour titre "Contre la frivolité " et rappelle l'intérêt sans exclusive que porte la revue au Nouveau Roman

J'appelle sérieux les livres écrits par des auteurs adultes et conscients, dont la seule ambition n'est pas d'ajouter une œuvre pathétique ou drôle à tant d'œuvres drôles et pathétiques. L'intérêt que certains d'entre nous portent au « nouveau roman » s'explique par là. Il ne signifie ni que nous adhérions à une conception déterminée de l'art romanesque ni que nous voulions ignorer les œuvres qui ne correspondent pas à ces canons. Mais il marque notre sympathie pour une littérature réfléchie, (p. 2-3)

Dans le même numéro figure un texte de Roland Barthes, " Le choix d'un métier », consacré au travail des femmes et publié dans une rubrique "Mythologies $»^{5}$. Une autre rubrique, "Le livre de la semaine », propose le compte rendu par Geneviève Serreau d'un livre dans lequel Edgar Morin revient sur son évolution politique et dont le titre, Autocritique, est assez parlant ${ }^{6}:$ «Peu ou prou beaucoup se reconnaîtront dans cette auto-analyse qui mêle à la confession l'examen passionné de l'Histoire et la remise en question des valeurs spirituelles sur lesquelles se fonde l'engagement révolutionnaire » ( $\mathrm{p}$. 11). Trois ans plus tard, Claude Simon publiera Le Palace, auquel il donnera pour exergue une définition du mot Révolution qui choquera certains à gauche. On peut lire aussi, dans la rubrique "Dans le monde des lettres ", une brève qui ne manque pas d'humour, sur les rapports entre roman (ancien et nouveau) et politique

\section{Balzac mal vu à Budapest}

En France, nous avons déjà eu l'occasion de le signaler, le "réalisme socialiste " est mort et enterré. Il est même de mauvais goût d'y faire allusion devant M. Pierre Daix. Et les meilleures places, à la prochaine vente du C.N.E. ${ }^{7}$, seront réservées à nos amis : Nathalie Sarraute, Claude Simon, Michel Butor, Bernard Pingaud, Édouard Glissant, qui y signeront leurs œuvres.

\footnotetext{
${ }^{5}$ Le recueil portant ce titre est paru en 1957.

${ }^{6}$ Edgar Morin avait été exclu du Parti communiste en 1951. Son livre est édité par Julliard, éditeur de la revue, dans une collection baptisée elle-même " Les Lettres Nouvelles ».

7 Comité National des Écrivains, né de la Résistance et contrôlé par le Parti communiste.
} 
Une information qui nous vient de Budapest ${ }^{8}$, et que publie le Monde du 29 mars, nous laisserait croire que là-bas on a plus de mal à prendre le tournant. Citons :

«L'attitude révisionniste de Georges Lukacs, écrit la revue Kortars (organe littéraire du parti socialiste ouvrier hongrois), a empêché la mise en œuvre des idées socialistes dans la littérature hongroise. Au lieu de reconnaître la grandeur des écrivains soviétiques, qui mettent en application d'une manière magistrale le réalisme socialiste, il a choisi Balzac et Tolstoï comme des exemples à suivre par les jeunes écrivains hongrois ».

Balzac ? Et Tolstoï ? Le criminel ! À Paris, quand Robbe-Grillet évoque Balzac c'est comme exemple à ne pas suivre. Tout formaliste et « jeune roman » qu'il soit, Robbe-Grillet est autrement plus communiste hongrois que cet odieux philosophe révisionniste, (p. 4).

Les grands écrivains, à la différence des médiocres, ne sont jamais en effet de mauvais modèles : il n'était pas inutile de le rappeler à Robbe-Grillet !

Le texte que Claude Simon donne aux Lettres nouvelles est, comme d'autres textes publiés en revue (par exemple "Matériaux de construction ", paru l'année suivante), un collage de fragments n'ayant pas de rapport explicite les uns avec les autres, et dont on peut supposer qu'ils ont été écrits séparément et assemblés pour l'occasion. Quatre fragments ici, sous un titre qui évoque le processus même de l'écriture et dont on entendra l'écho dans celui de la conférence prononcée douze ans plus tard par Simon à Cerisy : "La fiction mot à mot ». Mais si l'écriture est ainsi mise en avant, conformément à ce qui est déjà la marque de fabrique du Nouveau Roman, il n'en reste pas moins qu'elle ne fonctionne pas à vide, et que la densité de réel est frappante dans ces quatre fragments qui tous donnent au lecteur le sentiment de la « chose vue " (ou entendue). Certes, ce sentiment est construit par l'écriture, mais comment le serait-il à partir d'une expérience nulle ? Au contraire, affleure ici constamment un parti pris des choses au sens large du mot, incluant

\footnotetext{
${ }^{8}$ Rappelons que l'insurrection hongroise et l'intervention militaire soviétique se sont déroulées trois ans auparavant, en 1956.
} 
personnages et situations en tant qu'ils sont eux-mêmes réifiés par les conventions sociales qui les déterminent : le monologue d'une bourgeoise d'âge mûr déjeunant dans un restaurant, un enterrement dans un village espagnol, la conversation d'un couple revenant en autobus de Monte Carlo à Nice après avoir joué au casino, un rendez-vous d'affaire dans le bureau d'un négociant en vins du Midi.

Il s'agit donc dans chaque fragment d'une scène perçue, mais le dispositif de perception varie de l'une à l'autre. La scène du restaurant est tout entière représentée au discours direct, entre les points de suspension qui en laissent ouverts le début et la fin, comme un morceau d'enregistrement pris sur le vif. Le récit de l'enterrement suit le déroulement de la cérémonie selon le point de vue d'un observateur invisible et fasciné. La scène de l'autobus postule un passager témoin qui voit monter " ce couple incolore " (valeur déictique du démonstratif) et raconte l'échange en variant les formes du discours rapporté : direct, indirect, narrativisé. Enfin, la scène du bureau est la plus subjective puisque le narrateur est aussi l'un des personnages et qu'il fait état à la fois de ses perceptions et de ses réflexions. Cependant, les quatre fragments sont en quelque sorte unifiés par l'emploi dominant du présent, qui inscrit chaque scène dans le temps même de sa narration.

Si rien ne nous permet de remonter de ces quatre scènes à des circonstances particulières de la vie de Claude Simon, elles n'en restent pas moins marquées par une précision descriptive, un pouvoir d'évocation, qui laissent supposer une forte empreinte dans la mémoire, que l'écriture travaille à restituer. Dans le premier fragment, c'est la mimesis du discours oral qui est visée, en une sorte de sketch qui donne véritablement à entendre le sociolecte ridicule du personnage. Claude Simon montre là un talent d'imitateur corrosifqui le situe dans la lignée de Proust. Le second fragment évoque cette Espagne funèbre avec laquelle l'écrivain entretient une relation ambivalente - Le Palace, puis Histoire le montreront -, une Espagne à la fois proche et lointaine, objet de fascination et de répulsion, et qui se concentre ici dans le rituel doloriste des obsèques, la musique funèbre, le contraste violent entre le noir du deuil et la lumière du soleil. Le troisième fragment est comme un croquis saisi sur le vif au fil du voyage : deux silhouettes et quelques paroles volées suffisent à suggérer une forme de la misère humaine, avant d'être rendues au 
néant. Enfin, le dernier fragment pourrait se rattacher à l'activité de viticulteur qui est encore à l'époque celle de Claude Simon. Plusieurs indices suggèrent en effet que la scène se passe dans le bureau de quelque négociant auquel il aurait eu à vendre sa récolte. Et la « statue équestre d'un général coiffé d'un képi » se détachant sur « un fond de vignes et de montagnes » est probablement celle du maréchal Joffre, natif de Rivesaltes, dont la statue orne en effet la place centrale du village ${ }^{9}$. Et c'est bien l'atmosphère vivante d'un village du Roussillon dans les années cinquante qui est ici restituée - en contrepoint de celle, confinée, du bureau - avec ses " façades ocres », son " ciel trop bleu ", sa " lumière poussiéreuse ", ses platanes, ses cafés, ses gitans : comme un prolongement du Vent publié deux ans plus tôt.

Si les quatre scènes peuvent sembler disparates à première vue, il n'est pas impossible cependant de discerner entre elles des liens qui ont pu déterminer leur regroupement. La parole et l'argent, éléments essentiels de toute vie sociale, occupent en effet dans trois d'entre elles une place importante. La parole y est envisagée dans ce qu'elle a de vide et de stéréotypé, ne permettant aucune communication authentique (c'est bien sûr un grand thème des années cinquante, qui s'ouvrent avec La Cantatrice chauve de Ionesco) : logorrhée de la femme du restaurant, qui entrecoupe le récit d'une réception par des commentaires sur le déjeuner en cours, ou de la femme de l'autobus, autre bavarde qui ne laisse qu'un instant la parole à son mari et l'interrompt avant qu'il ait fini, et enfin dans la bouche du négociant " cette sorte de parade verbeuse qui se sert de termes de réclame et de prospectus ", comme un "rite " obligatoire en préambule à la négociation. Tel est donc aussi sans doute l'autre sens du titre : le vain " mot à mot " des parleurs comme objet de l'écriture. Quant à l'argent, il revient constamment dans les propos de la femme du

9 On se souvient de l'évocation féroce, dans L'Acacia, de ce chef militaire à la stratégie particulièrement meurtrière : "L'effectif était composé en majeure partie de recrues de ce département du Midi, bordé d'un côté par la mer et s'étendant à l'intérieur jusqu'aux premiers sommets des Pyrénées, dont était originaire le général en chef lui-même (l'homme corpulent - pas obèse : corpulent - aux grosses moustaches déjà blanches, vêtu d'un uniforme noir et d'une houppelande à pèlerine semblable à un camail de chanoine, et que les intrigues compliquées d'états-majors, de loges maçonniques et des salons du faubourg Saint-Germain avaient placé à la tête de l'armée en considération peut-être d'une placidité et d'une capacité de sommeil presque illimitée) [...]»(52). 
restaurant, estimant la valeur du buffet ou de l'hôtel, attentive aux signes extérieurs de richesse. On le retrouve dans la bouche de la passagère de l'autobus, obsédée par ses pertes au casino, et il est bien sûr le véritable enjeu du rendez-vous d'affaire. Reste le deuxième fragment, celui de l'enterrement, qui fait apparemment exception, si ce n'est qu'il décrit lui aussi un rituel social. Mais surtout, n'a-t-il pas pour fonction de rappeler ironiquement la vanité de l'existence humaine, qu'il s'agisse de la parole ou de la poursuite des biens matériels ? Quand la musique funèbre s'arrête, c'est pour accueillir le mort " dans les suaires déployés du silence »... L'humanité que décrit Claude Simon est donc à la fois médiocre, dérisoire et, parfois, pathétique, comme le suggèrent les images clôturant deux des fragments : une grosse femme portant péniblement une gerbe mortuaire sous le soleil écrasant, deux vieux joueurs qui ont encore perdu et qui «s'éloignent à petits pas, se tenant par le bras... ».

Des quatre fragments, le premier sera le seul repris ultérieurement par Claude Simon, aux pages 156 et 157 d'Histoire, publié en 1967. La scène sera alors intégrée à la journée qui sert de cadre temporel au roman, lorsque le narrateur déjeune dans un restaurant de la ville, observant et écoutant par intermittence cette femme que le serveur a installée à la table voisine, tout en errant dans ses souvenirs : Corinne (que l'âge, pense-t-il, a dû rendre proche de cette caricaturale bourgeoise), Barcelone, sa mère, l'oncle Charles. Le fragment sera donc contextualisé, mais il ne sera guère modifié. Le changement le plus important consistera dans la suppression de la ponctuation, générale dans Histoire, mais qui renforcera tout particulièrement ici l'effet de flux verbal continu et vain : « [...] des mots puis des phrases peut-être simplement alignés bout à bout dans l'unique raison de faire du bruit [...] " (Hist., 182). Quelques modifications mineures concerneront des références, par effacement ou au contraire précision : " une robe exquise de chez Dior » deviendra " une robe exquise de chez un grand couturier» (158), " une Frégate bleue " " une grosse voiture bleue ", "l'hôtel d'O. " " cet hôtel tu sais l'autre jour à Toulouse ». Les autres ne seront que des ajustements d'expression très ponctuels et peu significatifs. Simon aura donc récupéré et inséré dans son roman un texte écrit plusieurs années au- 
paravant ${ }^{10}$, ce qui confirme sa manière de travailler, qu'il expliquait lui-même ainsi lors de la sortie de L'Acacia : « J'écris "des choses" et j'espère qu'à la fin, ça pourra faire un livre... $\|^{11}$. «Des choses » en effet, qui souvent s'inspirent de « choses vues» restées imprimées dans la mémoire, et qui pourront tout aussi bien dormir dans quelque tiroir, être publiées dans les pages éphémères d'une revue, ou trouver leur place dans la vaste mosaïque d'un roman.

Jean-Yves LAURICHESSE

${ }^{10}$ Le monologue de la femme se prolonge dans Histoire aux pages 171-172 et 182-184, mais il est difficile de déterminer s'il était écrit tout entier dès 1959 ou s'il a été complété plus tard pour les besoins du roman.

"I Entretien avec Marianne Alphant, Libération, 31 août 1989. 